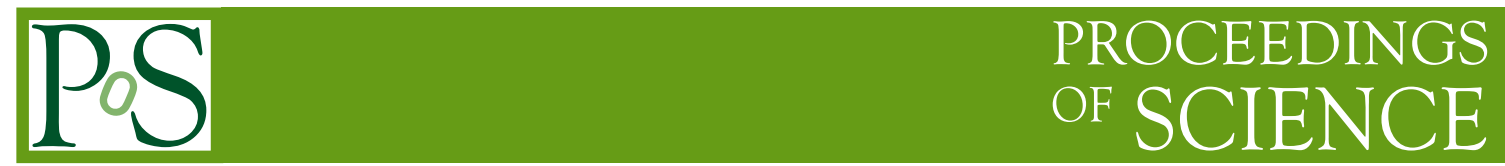

\title{
Recent Results from the XENON Experiment
}

\author{
Annika Behrens* ${ }^{\dagger}$ \\ University of Zurich \\ E-mail: behrens@physik.uzh.ch
}

The XENON100 experiment is a direct dark matter search experiment using a dual-phase xenon time projection chamber situated at the Laborati Nazionali de Gran Sasso (LNGS) underground laboratory in Italy. Due to its ultra-low background level it has been able to set competitive exclusion limits for the spin-independent and the spin-dependent cross-section for the scattering of weakly interacting massive particles (WIMPs) off nucleons. Its successor, XENON1T, is currently under construction, aiming to improve upon the sensitivity of XENON100 by two orders of magnitude.

The European Physical Society Conference on High Energy Physics

18-24 July, 2013

Stockholm, Sweden

\footnotetext{
* Speaker.

†n behalf of the XENON collaboration
} 


\section{XENON100}

While there are numerous hints to the existence of dark matter [1], its nature has yet to be determined. One main candidate that arises naturally in many theories beyond the standard model is the weakly interacting massive particle (WIMP) [2].

The XENON100 experiment [3] aims at detecting dark matter via the elastic scattering of WIMPs off xenon nuclei in a cylindrical dual-phase (liquid/gas) time-projection chamber (TPC). The target mass of $62 \mathrm{~kg}$ of liquid xenon is optically separated from an additional $99 \mathrm{~kg}$ of liquid xenon which is used as an active scintillation veto surrounding the TPC.

When a particle interacts with the xenon the latter becomes both excited and ionised. The prompt scintillation light (S1) is detected by two arrays of photomultiplier tubes (PMTs) at the top and the bottom of the TPC. The ionisation charge is drifted by an electric field towards the gas phase, where it is converted via proportional scintillation to a secondary light signal (S2), which is also observed by the PMTs. The ratio between $\mathrm{S} 1$ and $\mathrm{S} 2$ can be used to discriminate between signal and background events. Fiducialisation of the TPC volume is possible thanks to the full spacial position reconstruction, based on the drift time between the S1 and the S2 signal and the S2 hit pattern on the PMTs.

\section{Recent Results}

The 2011/2012 science run of XENON100 [4] consisted of 224.6 live days of dark matter data. Data taking was interrupted only for weekly calibrations with LED light for monitoring the PMTs and ${ }^{60} \mathrm{Co}$ and ${ }^{232} \mathrm{Th}$ sources to study the electronic recoil (ER) response of the detector. For the nuclear recoil (NR) response, a calibration with an AmBe source has been performed at the beginning and at the end of the run.

The benchmark WIMP search region was defined from $3-20$ PE in S1 $(6.6-30.5 \mathrm{keV}$ nr), with an upper $99.75 \%$ ER rejection line and a lower line at $\sim 97 \%$ NR acceptance in the discrimination parameter space. Data was blinded during data taking in the region of $2-100$ photoelectrons (PE) in $\mathrm{S} 1$ in order to avoid analysis bias.

To estimate the ER background the ${ }^{60} \mathrm{Co}$ and ${ }^{232} \mathrm{Th}$ calibration data, with more than 35 times the statistics in the dark matter search data, was scaled to match the non-blinded side band. In the benchmark region $(0.79 \pm 0.16)$ events from ER were expected. The NR background expectation of $\left(0.17_{-0.07}^{+0.12}\right)$ events has been determined by Monte Carlo simulations using precise measurements of the radioactive contamination of all detector materials and the muon energy and angular dependance at LNGS. The total background expectation in the benchmark region was (1.0 \pm 0.2$)$ events.

After unblinding, 2 events were observed in the benchmark region (Fig. 1), with energies of $7.1 \mathrm{keV}_{n r}$ and $7.7 \mathrm{keV}_{n r}$, both in the lowest energy bin included in this analysis. The waveforms of both events were of high quality. The profile likelihood analysis yielded a p-value of $\geq 5 \%$ for all WIMP masses indicating no excess due to a dark matter signal. The probability for the expected background in the benchmark region to fluctuate to 2 events is $26.4 \%$, which confirms the background-only hypothesis.

Using standard assumptions of an isothermal WIMP halo with a local density of $\rho_{\chi}=0.3 \mathrm{GeV} / \mathrm{cm}^{3}$, a local circular velocity of $v_{0}=220 \mathrm{~km} / \mathrm{s}$ and a galactic escape velocity of $v_{e s c}=544 \mathrm{~km} / \mathrm{s}$ an ex- 


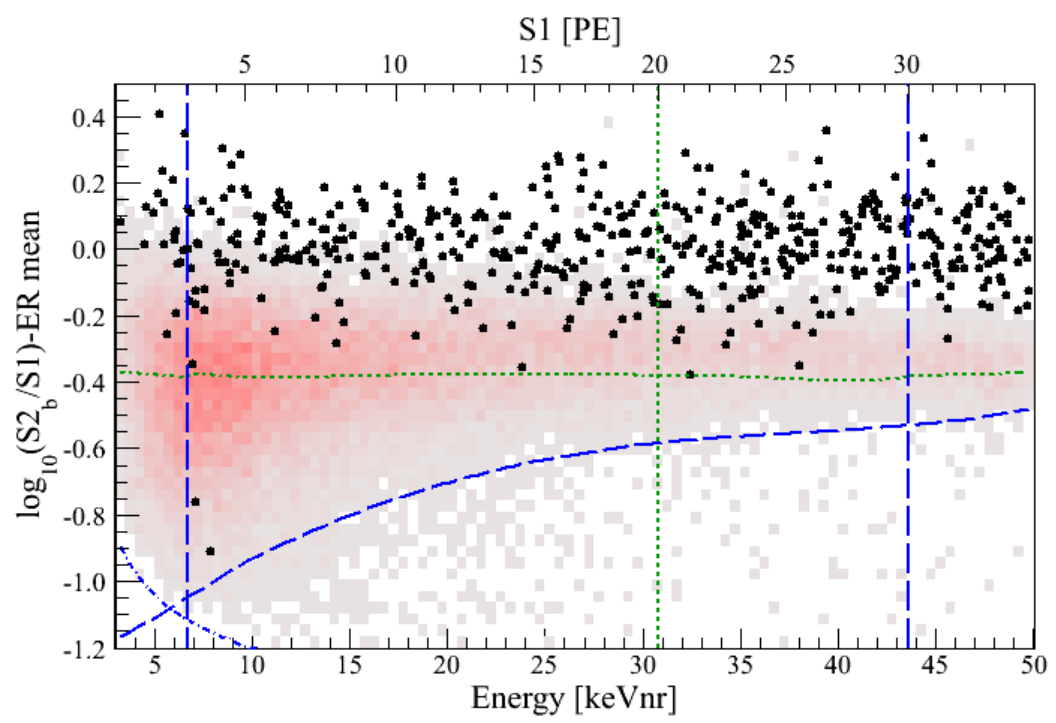

Figure 1: Event distribution in 225 live days after unblinding. Indicated is the WIMP search region in the flattened discrimination space, defined by a lower analysis threshold at $3 \mathrm{PE}$, a lower border along $97 \%$ NR acceptance (dashed blue), an upper border at $99.75 \%$ ER rejection (dotted green) and an S2 threshold at 150 PE (dashed-dotted blue). There are two valid invents within this benchmark region. The red-gray histogram shows the NR band obtained from the AmBe calibration.

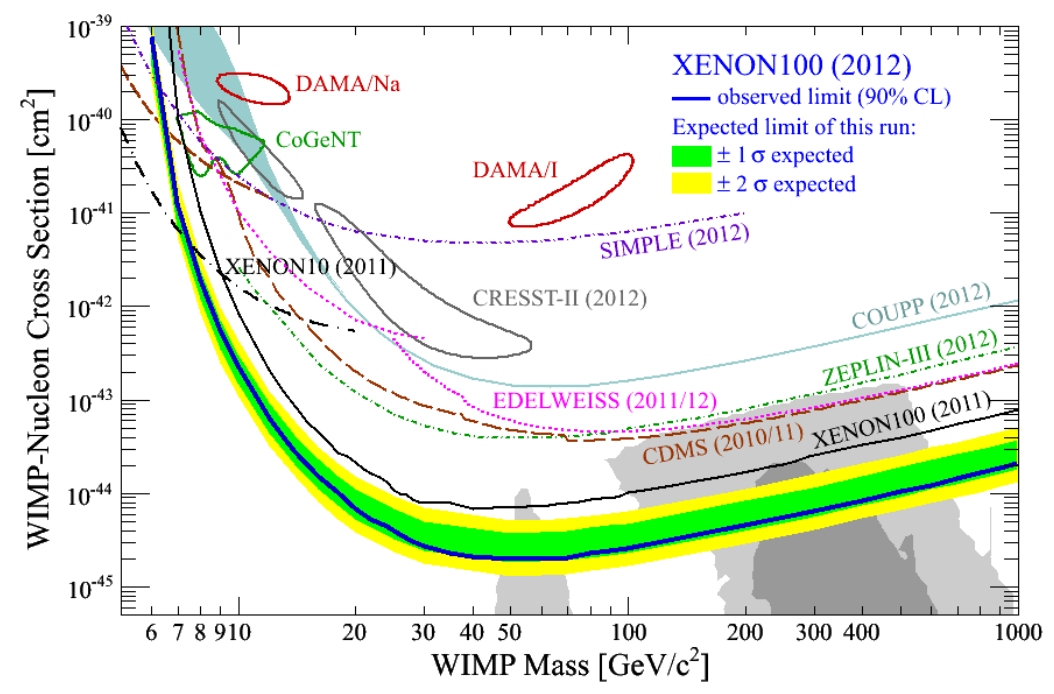

Figure 2: Exclusion limit for spin-independent WIMP-nucleon coupling from 225 live days from XENON100 in comparison with the results from other direct dark matter searches. 


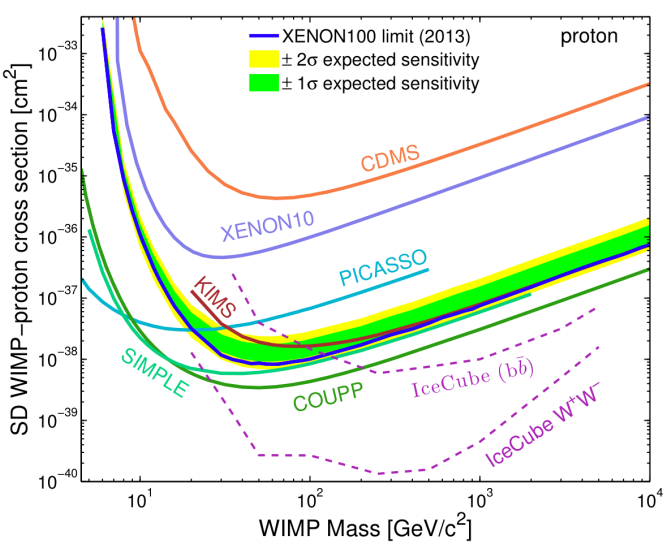

Figure 3: WIMP exclusion limit from 225 live days for spin dependent coupling to protons.

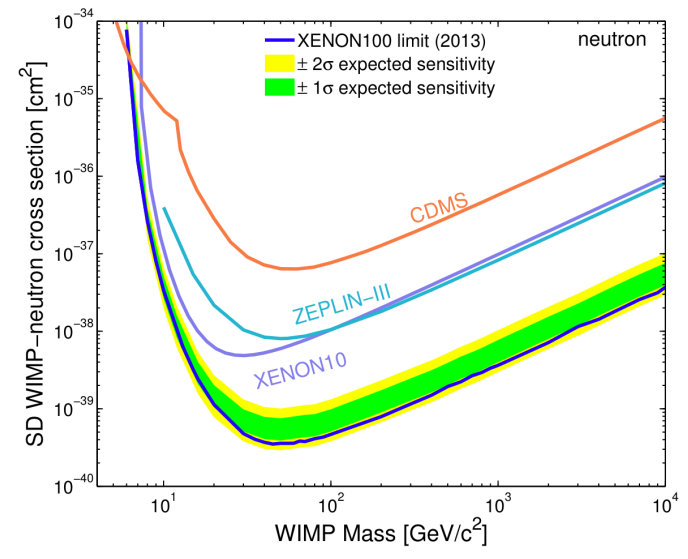

Figure 4: WIMP exclusion limit from 225 live days for spin dependent coupling to neutrons.

clusion limit for spin-independent WIMP-nucleon cross sections $\sigma_{\chi}$ has been calculated at $90 \%$ confidence level using the profile likelihood method (Fig. 2). Systematic uncertainties in the energy scale as described by the $\mathscr{L}_{\text {eff }}$ and in the background expectation are profiled out.

The existence of xenon isotopes with non-zero spin, namely ${ }^{129} \mathrm{Xe}$ (spin 1/2) with an abundance of $26.2 \%$ and ${ }^{131} \mathrm{Xe}$ ( $\operatorname{spin} 3 / 2$ ) with an abundance of $21.8 \%$ in the XENON100 detector also allows for a spin-dependent analysis. The nuclear shell model by Menendez et al. [6] was used for the structure function. Analogue to the spin-independant analysis exclusion limits have been set [5], both for WIMP-proton coupling (Fig. 3) and WIMP-neutron coupling (Fig. 4).

\section{Nuclear Recoil Energy Scale}

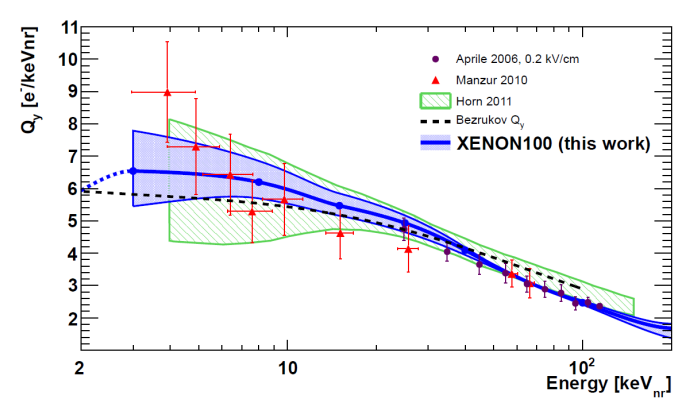

Figure 5: $\mathscr{Q}_{y}$ as obtained from matching Monte Carlo simulations to calibration data.

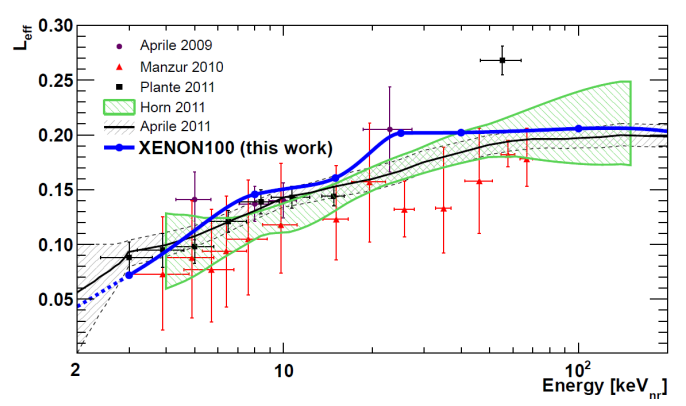

Figure 6: $\mathscr{L}_{\text {eff }}$ as obtained from matching Monte Carlo simulations to calibration data.

The data taken with an AmBe source has been compared to Monte Carlo simulations in order to verify the energy scale that has been used for the dark matter search results [7]. A detailed description of the detector, including the shield, was used in modelling the S1 and S2 response. In a first step, the S2 signal was matched using the $\mathscr{L}_{\text {eff }}$ derived from direct measurements, determining the charge yield $\mathscr{Q}_{y}$ (Fig. 5). Best results were achieved assuming a neutron rate of $159 \mathrm{n} / \mathrm{s}$, which is in agreement with the independent measurement of the source strength of $(160 \pm 0.4) \mathrm{n} / \mathrm{s}$. 
In a second step, using the derived $\mathscr{Q}_{y}$, the $\mathrm{S} 1$ signal has been similarly matched, yielding a parameterisation of $\mathscr{L}_{\text {eff }}$ (Fig. 6). For both the $\mathrm{S} 1$ and the $\mathrm{S} 2$ spectrum, the Monte Carlo simulation and the calibration data are in very good agreement, confirming the energy scale used in the dark matter search.

\section{XENON1T}

The successor of XENON100 is XENON1T, which is currently under construction at LNGS and expected to begin commissioning by the end of 2014. XENON1T will use a target of $2.2 \mathrm{t}$ of liquid xenon and is designed to improve upon the sensitivity of XENON100 by two orders of magnitude (Fig. 7), with a background expectation of less than one event in a total exposure of $2 \mathrm{t} \cdot \mathrm{y}$. To achieve this, all detector material will be carefully selected for low-radioactivity, the contamination with krypton and radon will be reduced, and a water muon veto will be used.

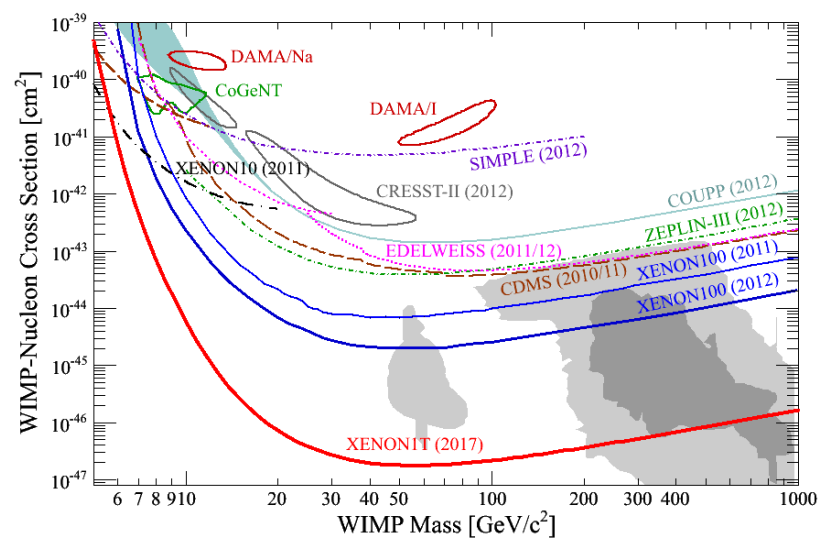

Figure 7: Projected sensitivity for XENON 1T.

\section{References}

[1] G. Bertone et al., Particle Dark Matter: Evidence, Candidates and Constraints, Phys. Rep. 405, 279 (2005), [hep-ph/ 0404175$]$

[2] G. Jungman et al., Supersymmetric Dark Matter, Phys. Rept. 267, 195-373 (1996), [hep-ph/9506380]

[3] E. Aprile et al. (XENON100),The XENON100 Dark Matter Experiment, Astropart. Phys. 35, 573-590 (2012), [1107.2155]

[4] E. Aprile et al. (XENON100), Dark Matter Results from 225 Live Days of XENON100 Data, Phys. Rev. Lett. 109, 181301 (2012), [1207.5988]

[5] E. Aprile et al. (XENON100), Limits on spin-dependent WIMP-nucleon cross sections from 225 live days of XENON100 data, Phys. Rev. Lett. 111, 021301 (2013), [1301.6620]

[6] J. Menendez et al., Spin-dependent WIMP scattering off nuclei, Phys. Rev. D 86, 103511 (2012), [1208.1094]

[7] E. Aprile et al. (XENON100), Response of the XENON100 Dark Matter Detector to Nuclear Recoils, Phys. Rev. D 88, 012006 (2013), [1304 .1427] 